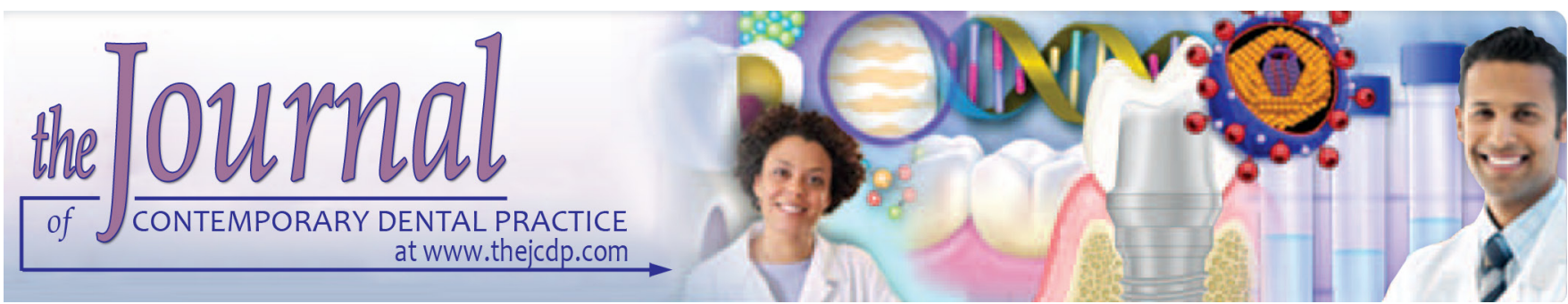

\title{
Analysis of Vertical Misfit of Crowns Fabricated with CAD/CAM Technology using Two Scanning Techniques: Direct and Indirect
}

\author{
${ }^{1}$ Wilson M Junior, ${ }^{2}$ Cleuber R de S Bueno, ${ }^{3}$ José R de AC Filho, ${ }^{4}$ Luciane SA Osorio, ${ }^{5}$ Maria C Neves, ${ }^{6}$ Joel FS Junior, \\ ${ }^{7}$ Hugo N Filho
}

\begin{abstract}
Aim: The study evaluated the marginal vertical misfit of feldspathic ceramic crowns fabricated by the computer-aided design (CAD)/computer-aided manufacturing (CAM) technology and compared the two methods of scanning techniques: direct digital impression and indirect digital impression.
\end{abstract}

Materials and methods: The titanium specimens were divided into two groups: scanning with the direct digital impression (DDI) at the milled prosthetic abutment level and indirect digital impression (IDI) at the cast model and after milled feldspathic ceramics blocks. Vertical marginal misfit was analyzed. The t-test was used for the analysis of the comparison factor between the groups and the one-way analysis of variance (ANOVA) test, and post hoc Tukey test was used to compare the variance of crown analysis regions within the group. A significance level of $5 \%$ was considered for the analyses.

Results: There was no significant difference in vertical marginal misfit between the groups of DDI and IDI ( $p=0.345)$. In relation to each region measured within the studied groups, it was observed the similarity between the six regions analyzed in the DDI group $(p>0.05)$ and IDI group, a significant difference between two areas.

Conclusion: The vertical marginal adaptation was similar between digital scanning methods. Based on the data evaluated, vertical marginal adaptation indices were within acceptable clinical standards.

Clinical significance: The direct digital scanning in unit bodies was reliable, pointing that the coating of titanium dioxide in the titanium abutment did not negatively influence the vertical marginal adaptation of the feldspathic ceramic crowns.

Keywords: Computer-aided design/computer-aided manufacturing, Dental crown, Digital impression, Marginal gap.

${ }^{1-7}$ Department of Health Sciences, Universidade do Sagrado Coração, Bauru, Sao Paulo, Brazil

Corresponding Author: Cleuber R de S Bueno, Department of Health Sciences, Universidade do Sagrado Coração, Bauru, Sao Paulo, Brazil, Phone:+551421077000, e-mail: cleuberrsbueno@gmail.com
How to cite this article: Junior WM, Bueno CRdeS, Filho JRdeAC, Osorio LSA, Neves MC, Junior JFS, Filho HN. Analysis of Vertical Misfit of Crowns Fabricated with CAD/ CAM Technology using Two Scanning Techniques: Direct and Indirect. J Contemp Dent Pract 2019;20(3):285-290.

Source of support: Nil

Conflict of interest: None

\section{INTRODUCTION}

The development in manufacturing CAD/CAM technology has resulted in great advances in oral rehabilitation, highlighting improved productivity, cost reduction, reduced clinical time, and better functional outcome in the long term. This is justified by various studies carried out in this field. ${ }^{1-4}$

Currently, ceramic crowns are popularly used in oral rehabilitation. Marginal adaptation is an important factor in the longevity of such restorations since mismatches in ceramic pieces can lead to the dissolution of the cementing agent, microleakage and retention of biofilm, leading to clinical failure. ${ }^{5}$ Some studies have suggested that an adaptation of up to $120 \mu \mathrm{m}$ is acceptable $^{6}$ while some other studies have suggested the adequate value to be up to $100 \mu \mathrm{m}$. There is a lack of consensus regarding the marginal adaptation value. However, a systematic review study showed that is possible to obtain a tooth-prosthesis gap of less than 80 $\mu \mathrm{m}$ with systems CAD/CAM. ${ }^{7}$ It should also be noted that the type of scanning may influence the vertical marginal adaptation of the prosthesis performed by CAD/CAM technology. ${ }^{8}$

Different scanning techniques for CAD/CAM are used in both the dental laboratory and the dental office. Lab scanners are widely used for indirect extraoral digitization of gypsum models. Intraoral scanners are becoming widespread in clinical dental practice for direct data capturing. There is a need to compare the precision 
of these methods. In this sense, studies should investigate the different types of scans, including the indirect digital impression and direct digital impression, to conduct in clinical decisions. ${ }^{9,10}$ The current scanners avoid the use of traditional molding, are convenient for the patient and involve complete digital workflow. ${ }^{11}$

However, the accuracy of this method needs to be proved, and factors such as coating film and direct digital impression capacity need to be adequately established in the literature. ${ }^{12,13}$ Recently, Alikhasi et al. ${ }^{14}$ reported that there is a requirement of further laboratory and clinical studies on scanning methods and their correlation with the traditional method.

Thus, our study aimed to analyze the vertical marginal adaptation of ceramic crowns obtained by means of two scanning techniques using the CAD/ CAM system. The null hypothesis of the work was that crowns made by the direct digital impression method would present similar indices of a vertical marginal misfit when compared to crowns made by the indirect digital impression method.

\section{MATERIALS AND METHODS}

\section{Standardization of Abutment}

For the standardization of the abutments and crowns, was performed the waxing of a mandibular right first molar following a previously established parameter ${ }^{15}$ and the scanning of its surface was carried out. The software Exocad $\AA$ (Dentalcad, Darmstadt, Germany) was designed as an intermediate for cemented prosthesis for the scanned inferior molar, standardized as follows: $10 \mathrm{~mm}$ (buccallingual), $12 \mathrm{~mm}$ (mesial-distal), $8 \mathrm{~mm}$ (height), with 1.2 $\mathrm{mm}$ of end line in chamfer and anatomical reduction of $1.5 \mathrm{~mm}$, and scanned using the Optimet DS6000 scanner (Optimet Phototonics, Israel) with standards and JIG recommended by the manufacturers the external hexagon implant with regular platform $(4.0 \mathrm{~mm})$ of the Original Bonelike (OBL; Rio Claro, São Paulo, Brazil) (Fig. 1). Using this model, 20 specimens of titanium were milled using the DM5 milling machine (Technodrill, Rio Grande do Sul, Brazil).

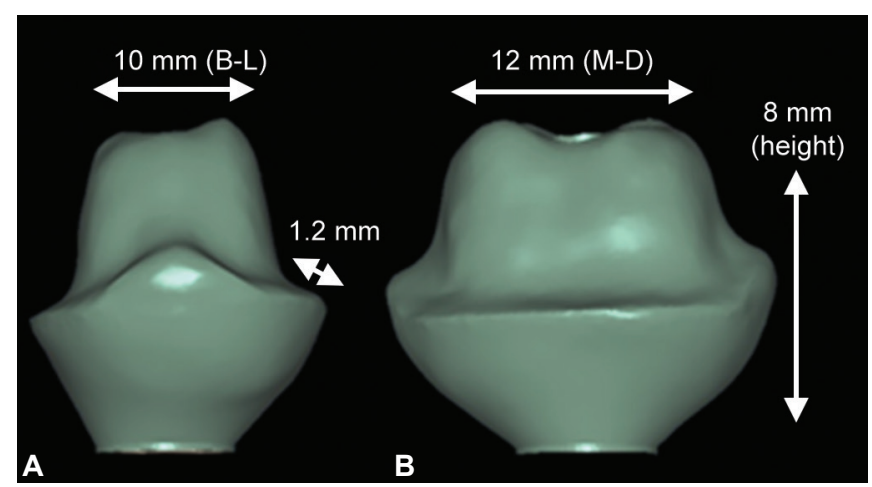

Figs $1 \mathrm{~A}$ and B: Standard design of the cemented abutment designed for the study

\section{Study Groups}

The study used two groups of 10 specimens. In the DDI) group, the titanium specimens (Fig. 2A) were scanned using the CEREC Scanner 3D Bluecam (Sirona Dental Systems $\mathrm{GmbH}$, Bensheim, Germany) via the titanium dioxide film coating procedure. All the scans were performed by the same operator at a standard distance of $3 \mathrm{~cm}$ for 5 seconds in oscillatory movements from buccal to lingual and mesial to distal (Fig. 2B). In the IDI group, 10 moldings of the titanium abutments were made with polyether (Impregum, 3M ESPE, Seefeld, Germany) with the aid of individual trays made from a thermoplastic material (Luxaform, DMG, Hamburg, Germany). The models were cast in type IV gypsum (Snow Rock, 3D Scan Stone, DK MUNGKO) (Fig. 2C). Scanning of the models was done using the Cerec Scanner 3D Bluecan (Sirona Dental Systems GmbH, Bensheim, Germany), without the coating of titanium dioxide film.

\section{Construction and Milling of Crowns}

All crown designs and cervical boundary delimitations were performed by the same operator using the CEREC software SW 4.2.4 (Sirona Dental Systems GmbH, Bensheim, Germany). The following configurations were used in the two groups: fixed adhesive attachment space: $80 \mu \mathrm{m}$ and thickness margin: $0 \mu \mathrm{m}$. The final design was sent to the In Lab MC XL CEREC (Sirona Dental Systems GmbH, Bensheim, Germany) milling unit which was used for the processing and milling of all crowns using CEREC feldspathic ceramic blocks (Sirona Dental Systems GmbH, Bensheim, Germany).

\section{Cementation of Crowns}

Each specimen was seated and gently pressed onto its respective abutment without prior adjustment. A light
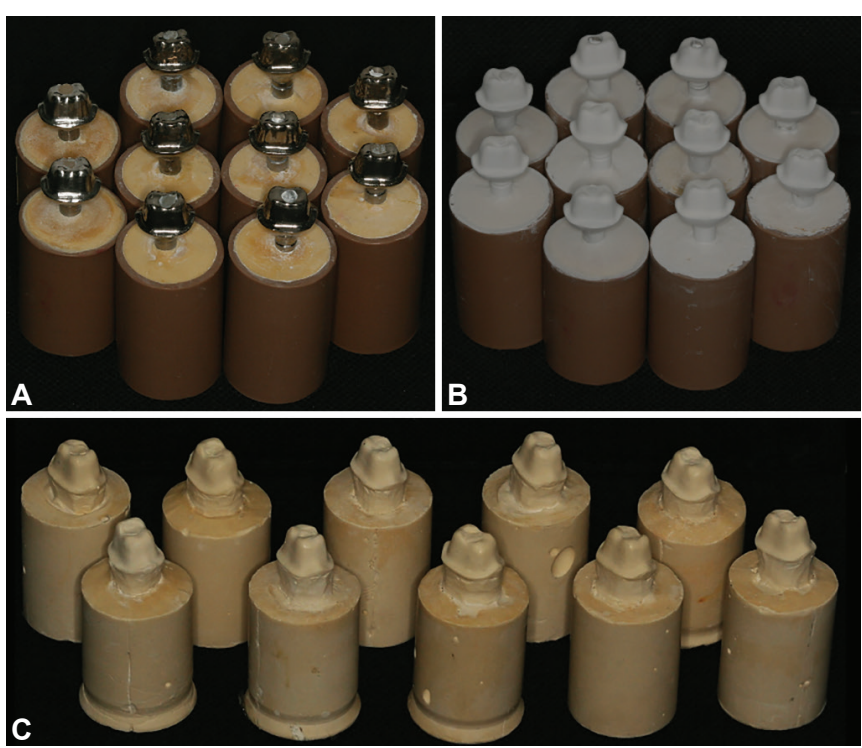

Figs 2A to C: (A) Titanium specimens of the DDI group before titanium dioxide film coating procedure; (B) After coating procedure; (C) The models of the group IDI that was scanned without coating procedure 
silicone addition pad (Virtual; Ivoclar Vivadent AG, Schaan, Liechtenstein) was placed inside each specimen. In order to standardize the applied force on the ceramic pieces, a metallic device with a fixed base was used, where the abutment and its respective specimen were positioned, and a movable one, in which a force of $50 \mathrm{~N}$ was applied, following protocol of the literature ${ }^{16}$ pointing exactly at the center of the ceramic crown, which waited for the setting time of the material indicated by the manufacturer.

\section{Analysis of Vertical Microgap}

Microscopic analysis of the interface of the abutment and crown was performed with the aid of a stereomicroscope (Olympus SZX9, Japan) coupled to a computer. They were placed perpendicularly at the base of the microscope and photomicrographs of the prosthetic interface were taken using the $42.8 \mathrm{x}$ objective. Through the program (ISCapture version 3.7.8, Xintu Phototonics, Tucsen, China), the specimens were measured. The six vertices of the platform of the external hexagonal implant were selected for the measurement of each specimen, exemplified in Figure 3A, and at each measurement point, 3 measurements of the prosthetic interface were made (Fig. 3B). Thus, there were a total of 18 measurements per specimen.

\section{Scanning Electron Microscopy}

Scanning electron microscopy of two randomly selected specimens was performed to compare the results of vertical marginal misfit using the electron microscopic EVO LS15 (Zeiss, Oberkochen, Germany) with a magnification of 50 and 100x.

\section{Statistical Analysis}

The obtained quantitative data were analyzed using Excel spreadsheets (Microsoft Office Excel, Redmond,
WA, United States), through the distribution of normality (Shapiro-Wilk) and variance (Brown-Forsythe). The t-test was used to analyze the comparison factor between the groups and respective regions (1-6) of the analysis of the crown between the two groups. To compare the effect of the mismatch for the different regions within each group, the one-way ANOVA test and post hoc Tukey test were used. The statistical program used was Sigma Plot 13.0 (San Jose, CA, USA), and a significance level of $5 \%$ was considered.

\section{RESULTS}

\section{General}

The vertical marginal adaptation of feldspathic ceramics and milled abutments showed no significant difference between IDI $(24.85 \pm 7.44 \mu \mathrm{m})$ and DDI $(24.06 \pm 8.54 \mu \mathrm{m})$ groups, and the data indicated similarity $(p=0,345)$. It was found that the DDI group had a lower median and lower dispersion of the data compared to the IDI group, as shown in Graph 1. The interquartile rates (25-75\%) of the vertical marginal adaptation for the printing systems were $23.13 \mu \mathrm{m}, 25.95 \mu \mathrm{m}$ for the IDI and $23.23 \mu \mathrm{m}, 24.42$ $\mu \mathrm{m}$ for the DDI, respectively.

The scanning electron microscopy analysis demonstrated the similarity in vertical marginal misfit for the two groups. It is important to note that milling can be verified with the respective details in the images. It is also possible to visualize the cementation in the indirect digital impression group (Fig. 4).

\section{By Region}

In relation to each region measured within the studied groups, we observed a similarity between the six regions analyzed in the DDI group $(p>0.05)$. However, in the IDI group, we observed a significant difference between areas D and $\mathrm{E}$. The medians, interquartile (25\% and $75 \%$ ), and major and minor points are expressed in Tables 1 and 2.
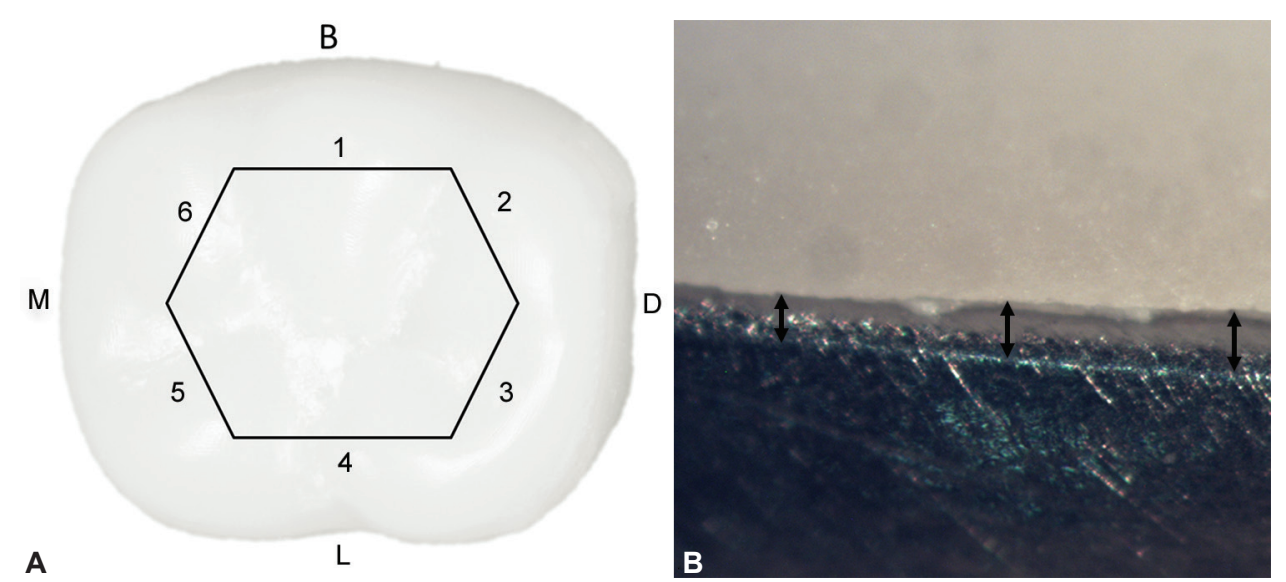

Figs $3 A$ and $B:(A)$ Methodology used to measure the microgap vertical and $3 b$, photomicroscopy $(42.8 x)$ demonstrating the reading of the three points of the vertical microgap 


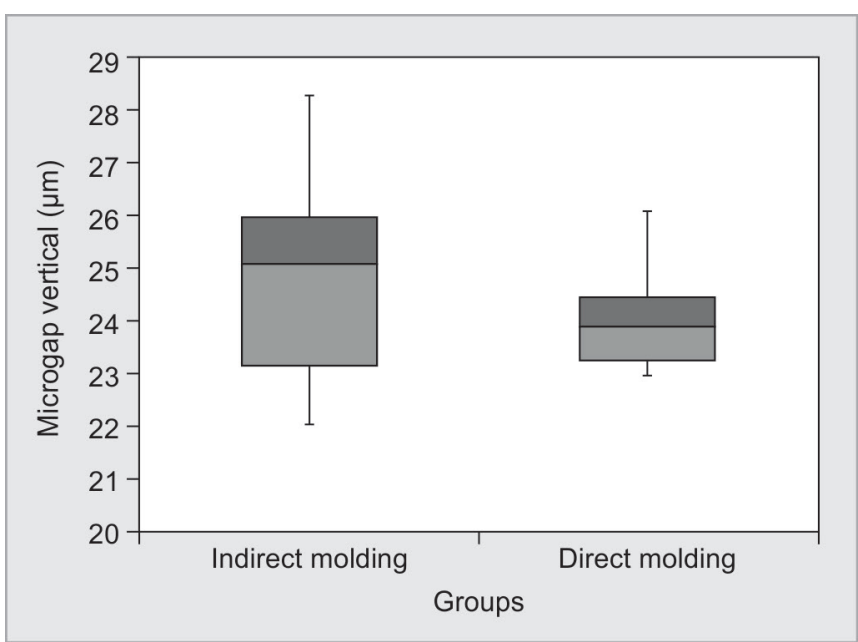

Graph 1: Box-plot graphic showing the median marginal gap of groups of the study (DDI and IDI) from highest to lowest and interquartile rates $(25 \%-75 \%)$

\section{DISCUSSION}

It was observed that vertical marginal adaptation was similar between digital scanning methods, which were within the standards clinically found in the literature. Therefore, the null hypothesis was accepted. The direct digital scanning in unit bodies was reliable, pointing that the coating of titanium dioxide in the titanium abutment did not negatively influence the vertical marginal adaptation of the feldspathic ceramic crowns. Corroborating with the literature, it has demonstrated that intraoral scanning is reliable in single pieces, and its limitation of evidence is linked to the scanning of
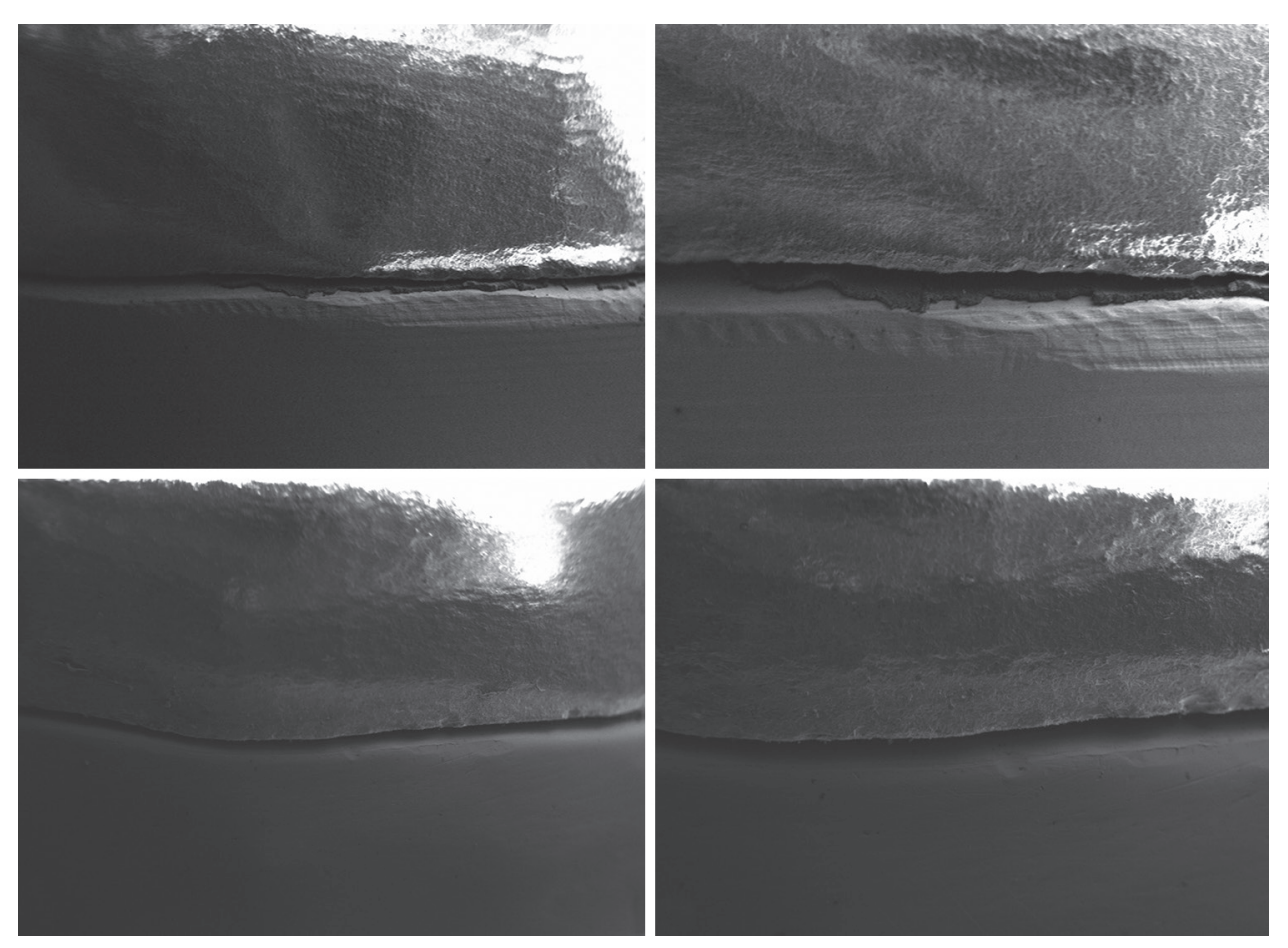

Fig. 4: Scanning electron microscopic (50 and 100x) of the groups study demonstrated similar microgap vertical marginal. 5a (50x) and $5 b(100 x)$, group IDI showing misfit vertical marginal, detail of the cement in the microap. 5c (50x) and 5d (100x), group DDI showing misfit vertical marginal

total arcs. ${ }^{12,17}$ In this study, although the cerec bluecam intraoral scanner was made for the direct digital method, the cerec bluecam was also used for the indirect method instead of a lab scanner because the central objective was actually to analyze the different techniques of digital scanning, therefore, if we used different scanners, we would be adding more variables in the study.

Analyzing the six regions measured, we observed similarities between all points in the direct digital impression group. In the indirect digital impression group, a significant difference between the two points was identified. This analysis can help determine whether there are balance and adaptation throughout the entire extent of the restoration. Although not influencing the overall mean vertical marginal adaptation, these differences may impact the passivity of the restorations. A critical factor that may influence these results is the delineation of the cervical preparation terminus line. ${ }^{18}$ Moreover, the operator experience is also considered essential, and the experience of the operative is essential because it is possible to finally correct some imperfection of the terminus line and scanning. Another point that may justify the results of the indirect digital impression group is related to the scanning being performed in a study model, which included a greater number of steps when compared to the other group. In our study, only one operator was responsible for the work, which reduced the chances of bias.

The vertical marginal adaptation is highly examined in in vitro tests due to its clinical relevance as vertical 
Analysis of Crowns with Two Scanning Techniques

\begin{tabular}{lllllll}
\hline \multicolumn{5}{c}{ Table 1: Vertical microgap results of each region measured DDI group } \\
\hline Region & Mean $(\mu \mathrm{m})$ & Standard deviation & Lowest value & Median & Highest value & Interquartile (25-75\%) \\
\hline 1 & 24.50 & \pm 8.76 & 17.45 & 22.34 & 62.52 & $20.12-26.60$ \\
2 & 23.55 & \pm 9.04 & 12.19 & 22.04 & 43.20 & $15.88-27.44$ \\
3 & 24.20 & \pm 11.55 & 10.07 & 21.18 & 62.35 & $18.34-26.16$ \\
4 & 26.06 & \pm 8.22 & 15.63 & 21.28 & 43.95 & $19.79-29.69$ \\
5 & 23.13 & \pm 7.87 & 13.98 & 21.23 & 43.09 & $17.08-26.88$ \\
6 & 22.95 & \pm 4.63 & 15.65 & 22.31 & 31.69 & $19.70-26.75$ \\
\hline
\end{tabular}

Table 2: Vertical microgap results of each region measured MI group

\begin{tabular}{lllllcc}
\hline Region & Mean $(\mu \mathrm{m})$ & $\begin{array}{l}\text { Standard } \\
\text { deviation }\end{array}$ & Lowest value & Median & Highest value & $\begin{array}{l}\text { Interquartile } \\
(25-75 \%)\end{array}$ \\
\hline 1 & 26.21 & \pm 7.05 & 15.67 & 28.04 & 35.24 & $20.21-31.95$ \\
2 & 24.91 & \pm 7.64 & 11.97 & 23.50 & 42.75 & $19.14-32.36$ \\
3 & 25.18 & \pm 8.35 & 14.01 & 23.29 & 40.71 & $19.77-30.97$ \\
4 & $28.28^{*}$ & \pm 7.87 & 10.87 & 27.45 & 48.05 & $25.38-30.55$ \\
5 & $22.02^{*}$ & \pm 5.37 & 9.76 & 23.16 & 30.87 & $19.06-25.66$ \\
6 & 22.54 & \pm 6.71 & 8.10 & 23.62 & 36.01 & $19.03-23.62$ \\
\hline
\end{tabular}

*Significant difference between groups $(p<0.05)$

faults can mechanically and biologically compromise restoration in the long term ${ }^{19}$ Larger misfit would be subject to complementation by the restorative cement, which may dissolve over time. In this context, there is still a lack of consensus on the clinically acceptable marginal adaptation. A recent systematic review study demonstrated rates of $120 \mu \mathrm{m}$ is an acceptable index. ${ }^{20}$ However, other authors have presented their doubts as to whether this value would be the most ideal. The $\mathrm{CAD} / \mathrm{CAM}$ systems have enabled these standards to be lowered ${ }^{7}$ reported that the average CAD/CAM systems allow less than 80 um of microgap in dental restorations. In our study, considering the limitations of an in vitro study and having the ideal conditions, we obtained satisfactory results in both groups $(<25 \mu \mathrm{m})$ when compared with those accepted in the literature.

Many authors use vertical marginal analysis using 4 locations (mesial, distal, buccal and lingual) for data collection, ${ }^{21}$ however, argued that analyzes with more measurement points could improve the capacity of the analysis. In our study, we used a methodology with six areas of analysis, which provides greater accuracy for the assessment of the mismatches between the restoration and abutment. $\mathrm{Ng}$ et al. ${ }^{22}$ presented a model with 8 points of measurement and justified its accuracy by using more points to improve the sensitivity of the test. However, the author argues even though, that some points not measured could present misfit that is not collected. Therefore, in view of the variety in methodologies, the comparisons should be made with caution since many factors can influence the adaptation of the restorations.

Thus, more studies should be carried out to find other methodologies for the analysis of vertical marginal adaptation. It is necessary that, in the future, the measurement of the entire surface of the crowns is used, not only by points, as suggested by Holmes et al. ${ }^{23}$ and $\mathrm{Ng}$ et al..$^{22}$ However, in view of our data, we must carry out in vivo studies using these protocols to evaluate the results clinically. We can verify the influence of intraoral factors such as saliva, humidity, and patient factors on intraoral scanner printing and thus, obtain better clinical conclusions.

\section{CONCLUSION}

Within the limitations of the present in vitro study, can be concluded that there was no statistically significant difference between the two scanning techniques (direct and indirect) in relation to vertical marginal maladaptation and coating with titanium dioxide did not influence the vertical marginal adaptation of the restorations in the direct scanning group.

\section{CLINICAL SIGNIFICANCE}

The vertical marginal adaptation using the two scanning techniques was considered within the clinical standards of the literature.

\section{ACKNOWLEDGMENT}

Authors are grateful to Institute P-I Brånemark, Bauru, São Paulo, Brazil for granting the use stereo microscopic and Universidade Estadual Paulista Filho, UNESP, Bauru, Brazil especially to technical Fabio Bossoi Vicente for granting and help to use a scanning electron microscope. Supported by grant number 15/14741-8 from the São Paulo Research Foundation (FAPESP).

\section{REFERENCES}

1. Katsoulis J, Müller P, Mericske-Stern R, et al. CAD/CAM fabrication accuracy of long- vs. short-span implant-supported FDPs. Clin Oral Implants Res 2015;26(3):245-249. 
2. Mello C, Santiago Junior JF, et al. Analysis of Vertical Marginal Adaptation of Zirconia Fixed Dental Prosthesis Frameworks Fabricated by the CAD/CAM System: A Randomized, Double-Blind Study. Int J Prosthodont 2016;29(2): 157-160.

3. de França DG, Morais MH, das Neves FD, et al. Precision Fit of Screw-Retained Implant-Supported Fixed Dental Prostheses Fabricated by CAD/CAM, Copy-Milling, and Conventional Methods. Int J Oral Maxillofac Implants 2017;32(3):507-513.

4. Al-Fouzan AF, Al-Mejrad LA, Albarrag AM. Adherence of Candida to complete denture surfaces in vitro: A comparison of conventional and CAD/CAM complete dentures. J Adv Prosthodont 2017;9(5):402-408.

5. Mounajjed R, Salinas TJ, Ingr T, et al. Effect of different resin luting cements on the marginal fit of lithium disilicate pressed crowns. J Prosthet Dent 2017.

6. McLean JW, von Fraunhofer JA. The estimation of cement film thickness by an in vivo technique. Br Dent J 1971;131: 107-111.

7. Boitelle P, Mawussi B, Tapie L, Fromentin O. A systematic review of CAD/CAM fit restoration evaluations. J Oral Rehabil 2014;41:853-874.

8. Dauti R, Cvikl B, Franz A, et al. Comparison of marginal fit of cemented zirconia copings manufactured after digital impression with lava ${ }^{\mathrm{TM}}$ C.O.S and conventional impression technique. BMC Oral Health 2016;16(1):129.

9. Haghi HR, Shiehzadeh M, Nakhaei M, et al. Effect of technique and impression material on the vertical misfit of a screw-retained, three-unit implant bridge: An in vitro study. J Indian Prosthodont Soc 2017;17(1):41-47.

10. Imburgia M, Logozzo S, Hauschild U, et al. Accuracy of four intraoral scanners in oral implantology: a comparative in vitro study. BMC Oral Health 2017;17(1):92.

11. Yuzbasioglu E, Kurt H, Turunc R, et al. Comparison of digital and conventional impression techniques: evaluation of patients' perception, treatment comfort, effectiveness and clinical outcomes. BMC Oral Health 2014;14:10.
12. Ahlholm P, Sipilä K, Vallittu P, et al. Digital Versus Conventional Impressions in Fixed Prosthodontics: A Review. Prosthodont 2016.

13. Basaki K, Alkumru H, De Souza G, et al. Accuracy of Digital vs. Conventional Implant Impression Approach: A ThreeDimensional Comparative In Vitro Analysis. Int J Oral Maxillofac Implants 2017;32(4):792-799.

14. Alikhasi M, Alsharbaty MHM, Moharrami M. Digital Implant Impression Technique Accuracy: A Systematic Review. Implant Dent 2017;26(6):929-935.

15. Guess PC, Zavanelli RA, Silva NR, et al. Monolithic CAD/ CAM lithium disilicate versus veneered Y-TZP crowns: comparison of failure modes and reliability after fatigue. Int J Prosthodont 2010;23:434-442.

16. Beuer F, Edelhoff D, Gernet W, et al. Effect of preparation angles on the precision of zirconia crown copings fabricated by CAD/CAM system. Dent Mater J 2008;27: 814-820.

17. Patzelt SB, Bishti S, Stampf S, et al. Fit of CAD/CAM implant frameworks: a comprehensive review. J Am Dent Assoc 2014;145(11):1133-1140.

18. Ribeiro IL, Campos F, Sousa RS, et al. Marginal and internal discrepancies of zirconia copings: effects of milling system and finish line design. Indian J Dent Res 2015;26(1):15-20.

19. Contrepois M, Soenen A, Bartala M, et al. Marginal adaptation of ceramic crowns: a systematic review. J Prosthet Dent 2013;110:447-454.

20. Shembesh M, Ali A, Finkelman M, et al. An In Vitro Comparison of the Marginal Adaptation Accuracy of CAD/CAM Restorations Using Different Impression Systems. J Prosthodont 2017;26(7):581-586.

21. Abdel-Azim T, Rogers K, Elathamna E, et al. Assessing the Fit of Implant Fixed Prostheses: A Critical Review. Int J Oral Maxillofac Implants 2010;25:506-515.

22. Ng J, Ruse D, Wyatt C. A comparison of the marginal fit of crowns fabricated with digital and conventional methods. J Prosthet Dent 2014;112(3):555-560.

23. Holmes JR, Bayne SC, Holland GA, et al. Considerations in measurement of marginal fit. J Prosthet Dent 1989;62(4):405-408. 